\title{
Progressive Concept Evaluation Method for Automatically Generated Concept Variants
}

\author{
Dereje Engida Woldemichael ${ }^{1, a}$ and Fakhruldin Mohd Hashim ${ }^{1}$ \\ ${ }^{1}$ Universiti Teknologi PETRONAS, Mechanical Engineering Department, Bandar Seri Iskandar, \\ 31750 Tronoh, Perak, Malaysia
}

\begin{abstract}
Conceptual design is one of the most critical and important phases of design process with least computer support system. Conceptual design support tool (CDST) is a conceptual design support system developed to automatically generate concepts for each subfunction in functional structure. The automated concept generation process results in large number of concept variants which require a thorough evaluation process to select the best design. To address this, a progressive concept evaluation technique consisting of absolute comparison, concept screening and weighted decision matrix using analytical hierarchy process (AHP) is proposed to eliminate infeasible concepts at each stage. The software implementation of the proposed method is demonstrated.
\end{abstract}

\section{Introduction}

Conceptual design process can be considered as design activity consisting problem definition, generation of concepts, firm up the generated concepts into concept variants followed by evaluation to decide the best concept for further development. Once the design space is explored exhaustively and possible concepts generated, the next major decision making process will be selection of the best concept variant. The decision made at this stage affects the subsequent design process. Hence, care must be taken not to overlook better design options, particularly when the concept generation process is done using automated concept generation process where large number of concept variants is generated. At the early stage of design, product concepts always need refinement and are subject to change. However, changes made later in the design stage are costly. Thus, exhaustive concept evaluation process should be implemented.

Concept selection is the process of evaluating concepts with respect to customer needs and other criteria, comparing the relative strengths and weakness of the concepts and selecting one or more concepts for further investigation, testing, or development [1]. Unfortunately, this decision is made at the stage where the designers have incomplete, uncertain, and evolving information about the concepts.

\footnotetext{
${ }^{a}$ Corresponding author : dereje.woldemichael@petronas.com.my
}

This is an Open Access article distributed under the terms of the Creative Commons Attribution License 2.0, which permits unrestricted use, distribution, and reproduction in any medium, provided the original work is properly cited. 


\section{Concept Selection Methods}

The most common formal and systematic methods of concept selection methods include Pugh's evaluation method (concept screening method as modified by [1]), weighted decision matrix, and analytical hierarchy process (AHP). In addition to these, there are less structured methods used in industry such as absolute comparison, concept review meetings, checklists or expert assessment (based on personal preference and expertise), voting on concept variants, and intuitive selection of concepts. The study conducted by [2] indicated that the degree of utilization of formal and systematic concept selection methods in industry is relatively low. On the other hand, the less structured methods possess a higher degree of utilization in the industry than the formal and systematic methods. However, the finding also concluded a higher degree of satisfaction in those companies using one or more formal and systematic concept selection methods compared to those companies who do not use. An overview of the systematic methods of concept selection approaches is presented next.

Pugh's Selection Method. Proposed by Stuart Pugh [3], this method is the most widely known and referred concept selection methodology, $[1,4-6]$. The evaluation process is performed by comparing each concept variant and the datum concept with respect to each criterion and giving values: "+" if the concept variant is better than the datum, "-" if the concept variant is worse than the datum, or " 0 " or " $\mathrm{S}$ " if the concept variant is same as the datum concept. A score pattern for each concept variant is calculated as the number of pluses, minuses and zeroes or S's. Even though this method can be used to eliminate infeasible concepts it assumes all the criteria are equally important and it did not indicate how much better or worse the concept is compared to the datum. Concept screening method [1] is a modified version of Pugh's concept selection method where the net score of the concept variants is calculated as a sum of pluses and minuses allowing ranking of the concept variants. However, this method also inherits the limitation of Pugh's concept selection method.

Weighted Decision Matrix. A decision matrix is a method of evaluating competing alternative concepts by ranking the selection criteria with weighting factors and rating the degree to which each concept variant meets the criterion. In reality the evaluation criteria markedly differ in terms of importance, thus a relative weight is assigned to each criterion. The relative weight can be assigned by using either of the following methods [7]:

i. The direct assignment technique: where the decision maker assign weights based on his/her experience using certain evaluation standards.

ii. Pairwise comparison: In this method each criterion is compared with all other criteria one at a time, and rated in comparison matrix. A given criterion is rated as ' 0 ' if it is less important than the other and as ' 1 ' if it is more important.

iii. Analytical Hierarchy Process (AHP): AHP can be used to assign weights to each criterion by comparing with each other like pairwise comparison method using, a 9 point scale known as fundamental scale of AHP [8] to make the comparison finer.

The decision matrix is prepared with the selection criteria and their respective weights in the first and second column of the matrix respectively; whereas the alternative concepts to be evaluated are displayed on the top of the matrix. Each concept variant is rated with respect to each criterion by using a 5-point scale when the knowledge about the criteria is not detailed and an 11-point scale when the information is more complete [5].

After all the concept variants are rated with respect to each criterion, regardless of the used scale the total score for each concept variant is calculated as the weighted sum of the concept variant's rating given by:

$$
R_{j}=\sum_{i=1}^{n} R_{i, j}=\sum_{i=1}^{n} w_{i} r_{i, j}
$$

Where:

$R_{i, j}=$ weighted score of concept $j$ for the $i^{\text {th }}$ criterion

$w_{i}=$ weighting factor for $i^{\text {th }}$ criterion 
$r_{\mathrm{i}, \mathrm{j}}=$ row rating of concept $j$ for the $i^{\text {th }}$ criterion

$n=$ number of criteria

$R_{j}=$ the total score of concept $j$

Table 1 Weighted decision matrix

\begin{tabular}{|c|c|c|c|c|c|c|c|c|c|}
\hline & \multicolumn{8}{|c|}{ Concept Variants (CV) } \\
\hline & & \multicolumn{2}{|c|}{$C V-1$} & \multicolumn{2}{|c|}{ CV -2 } & & & \multicolumn{2}{|c|}{$C V-j$} \\
\hline $\begin{array}{c}\text { Selection } \\
\text { Criteria }\end{array}$ & weight & Rating & $\begin{array}{c}\text { Weighted } \\
\text { score }\end{array}$ & Rating & $\begin{array}{l}\text { Weighted } \\
\text { score }\end{array}$ & . & - & Rating & $\begin{array}{l}\text { Weighted } \\
\text { score }\end{array}$ \\
\hline $\mathrm{C}-1$ & $w_{1}$ & $r_{11}$ & $R_{11}$ & $r_{12}$ & $R_{12}$ & & & $r_{l j}$ & $R_{l j}$ \\
\hline $\mathrm{C}-2$ & $w_{2}$ & $r_{21}$ & $R_{21}$ & $r_{22}$ & $R_{22}$ & & & $r_{2 j}$ & $R_{2 j}$ \\
\hline & . & . & & . & & . & . & . & \\
\hline $\mathrm{C}-\mathrm{n}$ & $w_{n}$ & $r_{n 1}$ & $R_{n 1}$ & $r_{n 2}$ & $R_{n 3}$ & . & & $r_{n j}$ & $R_{n j}$ \\
\hline Total score & & $R_{1}$ & & $R_{2}$ & & & & $R_{j}$ & \\
\hline
\end{tabular}

Analytical hierarchy process (AHP). Proposed by Thomas L. Saaty [8], this method is a structured multi-criteria decision making framework well suited for evaluation problems whose criteria have a hierarchical structure. In using AHP, both the criteria and the alternative concepts are pair wisely compared. AHP provides a diagnostic tool for assessing the consistency of the preference and reduces the bias on the decision maker. However, it is a relatively complex method with long decision process, especially when the number of alternative concepts is large with increased number of criteria requiring each alternative concept to be compared with all others for each criterion.

\section{Concept Evaluation Methods Implemented in CDST}

Conceptual design support tool (CDST) is a conceptual design support system developed to assist designers in performing conceptual design process of mechanical products [9-11]. CDST is developed to assist designers in performing the conceptual design process such as functional modeling (using standard vocabularies of functions), generating concepts (from database) and displaying on morphology chart, concept combination, and concept evaluation. The concept evaluation process takes the output of the concept combination phase (i.e., concept variants) and gives ranked concept variants as an output. The number of concept variants depends on the number of subfunctions in the functional structure and number of alternative concepts generated from the database for each subfunction. The automated concept generation process results in large number of concept variants which require a thorough evaluation process to select the best design. To address this problem, a progressive concept evaluation technique is proposed to eliminate infeasible concepts at each stage. Accordingly, three concept evaluation methods (i.e., absolute comparison, concept screening, and weighted decision matrix) are implemented. The software implementation is to automatically prepare the selection matrix, provide GUI to accept selection criteria from the user as input, and perform arithmetic calculation while rating. The concept variants are retrieved from the knowledge-base of CDST both in schematic and text format. Once the user rate the concepts based on their merits, the software can calculate the net score and rank the concepts based on the result to assist the user in decision making.

The main window of the concept evaluation process in CDST is shown in Figure 1. The concept evaluation process starts by identifying evaluation criteria. The user can select predefined evaluation criteria (default values) or give new evaluation criteria using criteria input window. Figure 2 shows the window that accepts criteria from the user.

The first evaluation process is using absolute comparison method. In this method concept variants are evaluated based on go/no-go screening of customer requirement, judgment of feasibility of the design, and assessment of technological readiness using absolute comparison window. The user decides whether to continue or reject each concept variant by choosing either yes or no on the absolute comparison window. The components of each concept variants can be viewed either textually or schematically by double clicking on the respective concept variant column in the absolute comparison 
window (Figure 3). Once the decision is made, the user uses "Refresh" pull down menu to eliminate those rejected concept variants. This allows the designer to significantly reduce the number of concept variants in the succeeding evaluation processes.
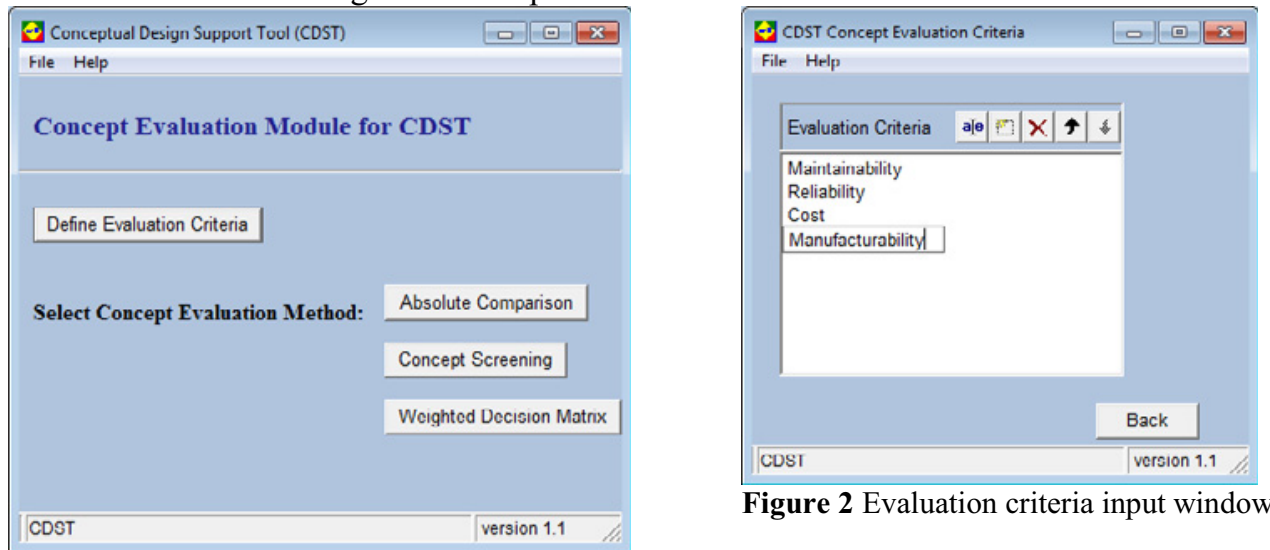

Figure 1 Concept selection process main window

Figure 2 Evaluation criteria input window

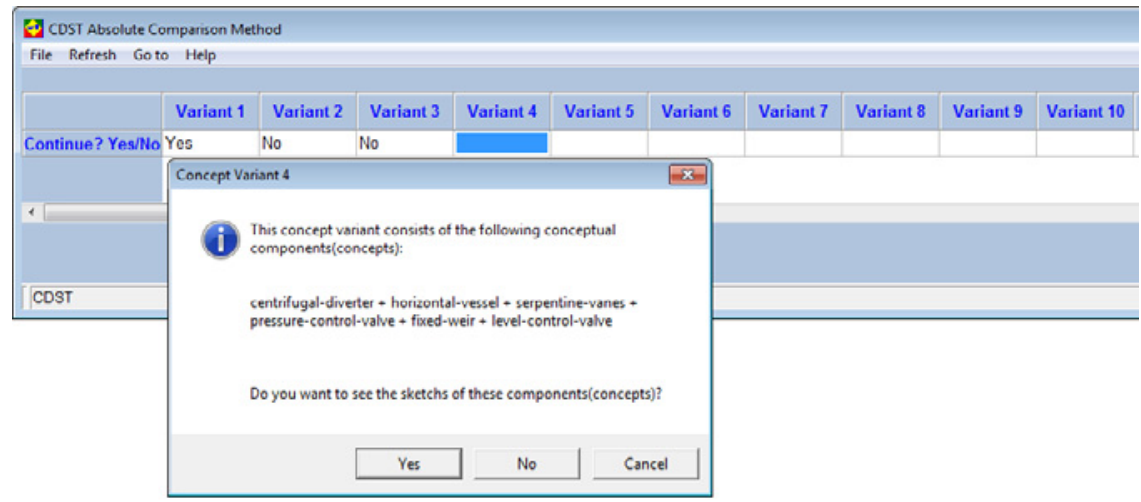

Figure 3 Absolute comparison window with list of concepts for concept variant 4

Next, the remaining concept variants are evaluated using concept screening method as shown in Figure 4. The concept screening window has pull down menu to select one of the concept variants or add a new concept variant as a datum. A datum concept variant is the one considered to be the best among the concept variants or a competitive products concept variant reduced to the same level of abstraction. After evaluation, the ranked concept variants are generated where the designer decides to reject the concept variants with lower ranking. This will further reduce the number of concept variants for final evaluation. 


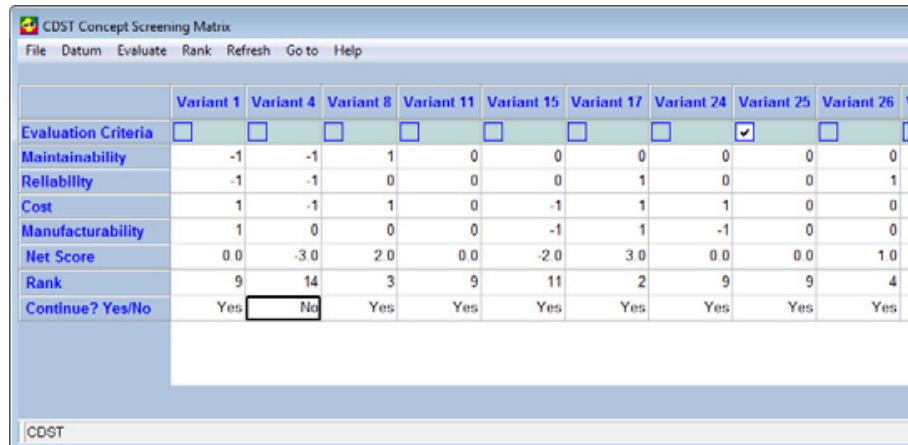

Figure 4 Screenshot of concept screening window

The remaining concept variants are finally evaluated by weighted decision matrix method. Here, the user chooses either a direct weight assignment or a pairwise comparison using AHP as shown in Figure 5. The pairwise comparison method to calculate the normalized weight is shown in Figure 6. Finally, the designer uses the weighted decision matrix and rate each concept variant using a 5-point scale or 11 point scale to get ranked concept variants as shown in Figure 7.

\section{Conclusions}

A progressive concept evaluation technique consisting of absolute comparison method from nonstructured concept selection method and concept screening and weighted decision matrix from structured and systematic concept selection methods were proposed to evaluate automatically generated concept variants. These methods were used to eliminate infeasible concept variants at each stage. The software implementation of these methods assists designers in handling repetitive and time consuming tasks.

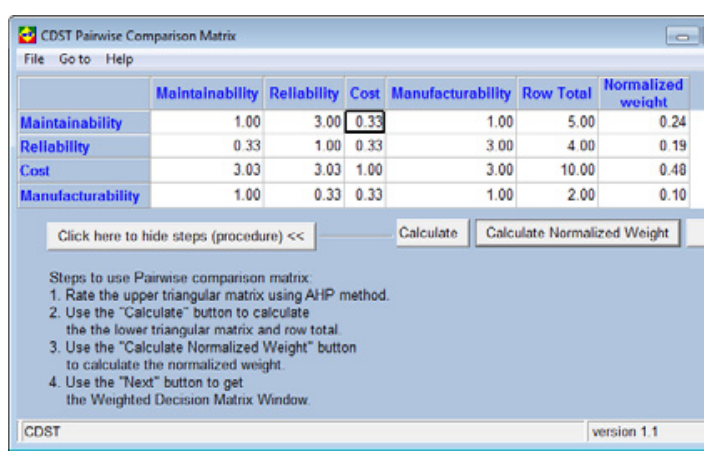

Figure 6 Pairwise comparison matrix

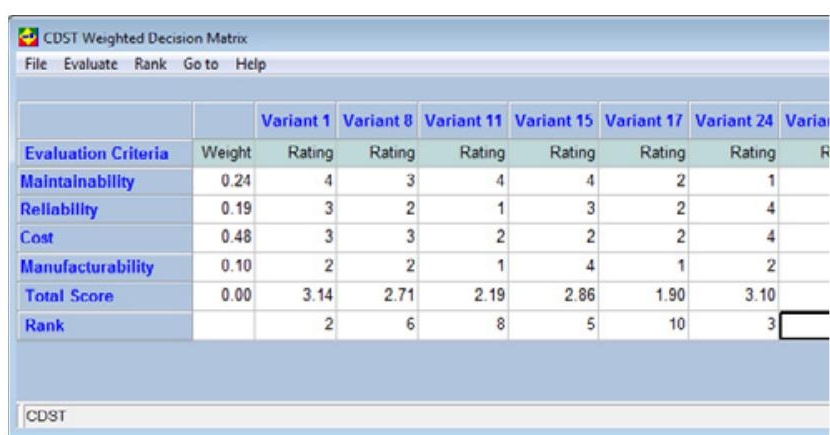

Figure 7 Final concept evaluation using weighted decision matrix

\section{References}

1. Ulrich, K.T. and S.D. Eppinger, Product Design and Development. $3^{\text {rd }}$ ed. 2004: McGRAWHill inc.

2. Salonen, M. and M. Perttula. Utilization of Concept Selection Methods - A Survey of Finnish Industry. in ASME 2005 International Design Engineering Technical Conferences and Computers and Information in Engineering Conference. 2005. Long Beach, California USA: ASME.

3. Pugh, S., Total Design: Integrated Methods for Successful Product Engineering. 1990: Addison-Wesley Publishing Company. 
4. Pahl, G. and W. Beitz, Engineering Design: A Systematic Approach. $2^{\text {nd }}$ ed. 1996, London: Springer.

5. Dieter, G.E., Engineering Design: A Materials and Processing Approach. $3^{\text {rd }}$ ed. 2000, New York McGraw-Hill.

6. Ullman, D.G., The mechanical design process. $3^{\text {rd }}$ ed. 2003: McGraw-Hill.

7. Sen, P. and J.-B. Yang, Multiple Criteria Decision Support in Engineering Design. 1998, New York: Springer.

8. Saaty, T.L., How to Make a Decision: The Analytic Hierarchy Process. Interfaces, 1994. 24(6): p. 19-43.

9. Woldemichael, D.E. and F.M. Hashim, Development of conceptual design support tool for subsea process equipment design. International Journal of Mechanical \& Mechatronics Engineering, IJMME, 2009. 9(10): p. 12-17.

10. Woldemichael, D.E., F.M. Hashim, and S. Debnath, A Knowledge-Based Conceptual Design Support System. Advanced Materials Research, 2011. 308: p. 1540-1545.

11. Woldemichael, D.E. and F.M. Hashim, A framework for function-based conceptual design support system. Journal of Engineering, Design and Technology, 2011. 9(3): p. 250-272. 